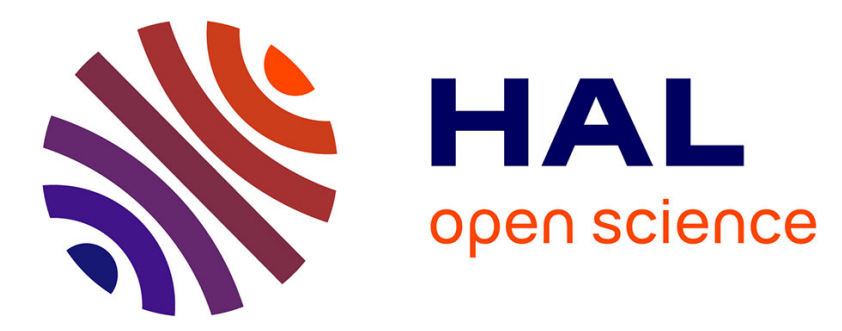

\title{
Capteur capacitif de petits déplacements. Application à la réalisation d'une balance de torsion enregistreuse de haute sensibilité
}

\author{
Daniel Chéry, Claude Grandjean, Jacques Villermaux
}

\section{To cite this version:}

Daniel Chéry, Claude Grandjean, Jacques Villermaux. Capteur capacitif de petits déplacements. Application à la réalisation d'une balance de torsion enregistreuse de haute sensibilité. Revue de Physique Appliquée, 1968, 3 (1), pp.5-10. 10.1051/rphysap:01968003010500 jpa-00242822

HAL Id: jpa-00242822

https://hal.science/jpa-00242822

Submitted on 1 Jan 1968

HAL is a multi-disciplinary open access archive for the deposit and dissemination of scientific research documents, whether they are published or not. The documents may come from teaching and research institutions in France or abroad, or from public or private research centers.
L'archive ouverte pluridisciplinaire HAL, est destinée au dépôt et à la diffusion de documents scientifiques de niveau recherche, publiés ou non, émanant des établissements d'enseignement et de recherche français ou étrangers, des laboratoires publics ou privés. 


\title{
GAPTEUR GAPAGITIF DE PETITS DÉPLAGEMENTS
}

\section{APPLIGATION A LA RÉALISATION D'UNE BALANGE DE TORSION ENREGISTREUSE DE HAUTE SENSIBILITÉ}

\author{
Par Daniel GHÉRY, Glaude GRANDJEAN et Jagques VillermauX, \\ Laboratoires de Génie Chimique de l'École Nationale Supérieure des Industries Chimiques, Université de Nancy, \\ Centre de Cinétique Physique et Chimique du C.N.R.S.
}

(Reçu le 12 octobre 196\%.)

\begin{abstract}
Résumé. - Un capteur capacitif est constitué par un condensateur variable de quelques picofarads pilotant la fréquence d'un oscillateur. Les variations de fréquence engendrent une tension continue proportionnelle au déplacement des armatures. Le capteur est utilisé pour détecter les rotations d'un fléau horizontal suspendu à un fil de torsion d'une cinquantaine de microns de diamètre. La microbalance ainsi constituée permet de mesurer et d'enregistrer des couples aussi faibles que $10^{-10}$ newton $\times$ mètre. Grâce à ce dispositif, on a pu mettre en évidence une " pression de succion " de $10^{-5}$ pascal, liée à l'adsorption irréversible d'atomes d'hydrogène sur l'une des faces d'une cible recouverte de trioxyde de molybdène.
\end{abstract}

Abstract. - A capacitive transducer has been devised which consists of a variable condenser of a few picofarads determining the frequency of an oscillator. Frequency variations are converted into a direct output voltage proportional to the relative displacement of plates. The transducer is used to monitor the rotation of an horizontal beam suspended by a torsion wire of about fifty microns diameter. With such a microbalance, torques as low as $10^{-1)}$ newton $x$ meter can be measured and recorded. By means of this device, evidence has been obtained for a "suction pressure" of $10^{-5}$ pascal, occuring when hydrogen atoms are irreversibly adsorbed onto a layer of molybdenum trioxide deposited on one side of a target.

L'étude de nombreux phénomènes physico-chimiques fait appel, directement ou indirectement, à la mesure et à l'enregistrement de faibles déplacements, c'est le cas notamment de toute une famille de micromanomètres à membrane décrits dans la littérature ou disponibles dans le commerce. Plusieurs dispositifs traducteurs ont été proposés et utilisés. Citons parmi d'autres les capteurs interférométriques, sans doute extrêmement sensibles, mais se prêtant mal à l'enregistrement, les capteurs potentiométriques, très simples et peu coûteux, mais peu fidèles en raison de l'existence d'un frottement solide, les capteurs à inductance variable, qui ont l'inconvénient de présenter une certaine inertie mécanique, et enfin les capteurs capacitifs. Une excellente revue d'ensemble sur ce type de capteurs a été publiée par Foldvari et Lion [1] et nous y renvoyons le lecteur pour plus de détails. La mesure se fait généralement en insérant la capacité variable dans un pont [2], [3], [4]. Lion [1], [5] propose en particulier un pont en double $\mathrm{T}$ qui présente une réponse linéaire autour du zéro. Le montage à résonance, où la capacité variable fait partie d'un circuit oscillant, permet en principe d'atteindre des sensibilités plus élevées, mais il a surtout été employé pour des mesures par une méthode de zéro [6]. Une réponse linéaire continue peut être obtenue en faisant battre l'oscillateur de mesure avec un oscillateur de référence [7], [8], avec l'inconvénient d'une complexité plus grande et la nécessité d'une symétrie et d'une stabilité particulièrement soignées. On peut concevoir un capteur à résonance à réponse continue en associant un discriminateur au circuit oscillant de mesure. Nous proposons ci-dessous deux montages de ce type et leur application à la détection des rotations d'une balance de torsion de haute sensibilité.

Le capteur électronique. - PRINGIPE DE FOnCtionNEMENT. - L'élément sensible du capteur est un condensateur variable $C$ placé en parallèle sur la capacité $C^{\prime}$ d'un circuit oscillant $\mathrm{L}-\mathrm{C}$ classique. Le déplacement à mesurer modifie la position relative des armatures métalliques de $\mathrm{C}$, en agissant soit sur l'écartement, soit sur la surface des armatures en regard, suivant la sensibilité et le domaine de linéarité désirés. Les variations de $\mathrm{C}$ font varier la fréquence de résonance $f=1 / 2 \pi \sqrt{L\left(C+C^{\prime}\right)}$ du circuit. Grâce à un montage électronique convenable, l'excursion relative en fréquence $\Delta f \mid f_{0}$ donne naissance à une tension 
de sortie continue qui lui est proportionnelle dans certaines limites. A condition de se borner à des déplacements d'amplitude limitée, on dispose donc d'une réponse linéaire. Le capteur peut fonctionner soit en appareil de zéro, soit en déviation autour d'une position donnée.

Le circuit L - G pilote un oscillateur stable de type Franklin qui fonctionne à une fréquence centrale constante $f_{0}$. La capacité $C^{\prime}$, grande devant $C$, comprend le câble de raccordement à la sonde de mesure et divers éléments de réglage et de compensation permettant de ramener la fréquence de résonance à $f_{0}$ quelle que soit $C$.

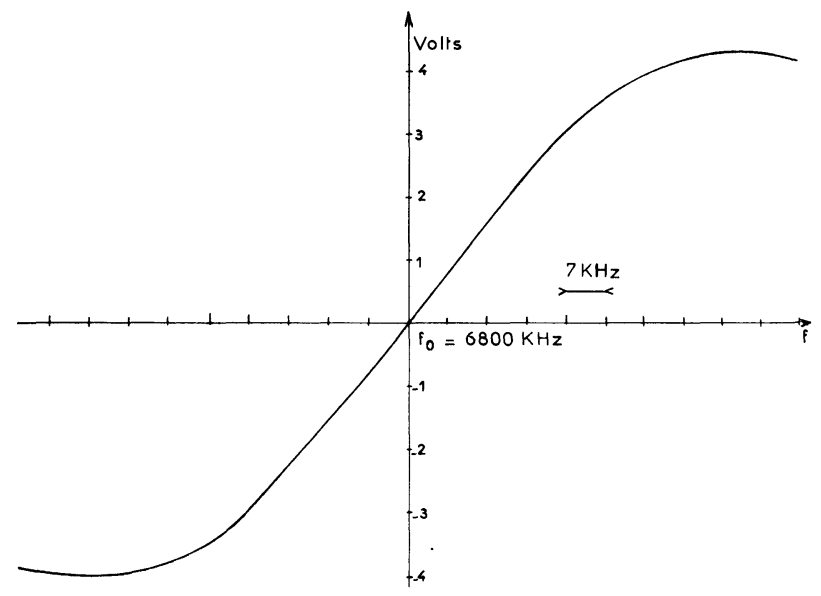

FIG. 1.
La tension de sortie de l'oscillateur, après amplification, est envoyée dans un discriminateur qui transforme les variations de fréquence en variations de tension. La caractéristique de démodulation de l'un de nos capteurs (version à tubes, schéma, fig. 2) est représentée sur la figure 1 . La linéarité est satisfaisante autour de la fréquence centrale, mais dépend d'une manière très critique du couplage entre le primaire et le secondaire, et de la symétrie de ce dernier enroulement. Un étage adaptateur sépare le discriminateur de la sortie.

RÉAlisations ET PERFORMANGES. - Un premier appareil de cette famille a déjà été décrit [20] et utilisé en association avec un micromanomètre à cuiller. Depuis, le montage a été considérablement amélioré en stabilité et en sensibilité. Deux versions en ont été construites. Les schémas détaillés en sont donnés respectivement par les figures 2 et 3 . Au maximum de sensibilité, le capteur à tubes délivre une tension de sortie de $750 \mathrm{mV}$ pour une excursion relative en fréquence de $10^{-3}$ autour d'une fréquence centrale d'environ $f_{0}=6,8 \mathrm{MHz}$. Le capteur transistorisé est un peu moins sensible. Pour une capacité totale $C+C^{\prime}=63 \mathrm{pF}$, la sensibilité est de $1,2 \mathrm{~V} / \mathrm{pF}$; la pente de la caractéristique est ainsi $150 \mathrm{mV}$ par $10^{-3}$ autour de la fréquence centrale $f_{0}=10 \mathrm{MHz}$.

Ces capteurs sont polyvalents et peuvent s'adapter à la mesure et à l'enregistrement de tout phénomène susceptible de modifier une capacité d'une dizaine de picofarads. Nous les avons surtout utilisés en liaison

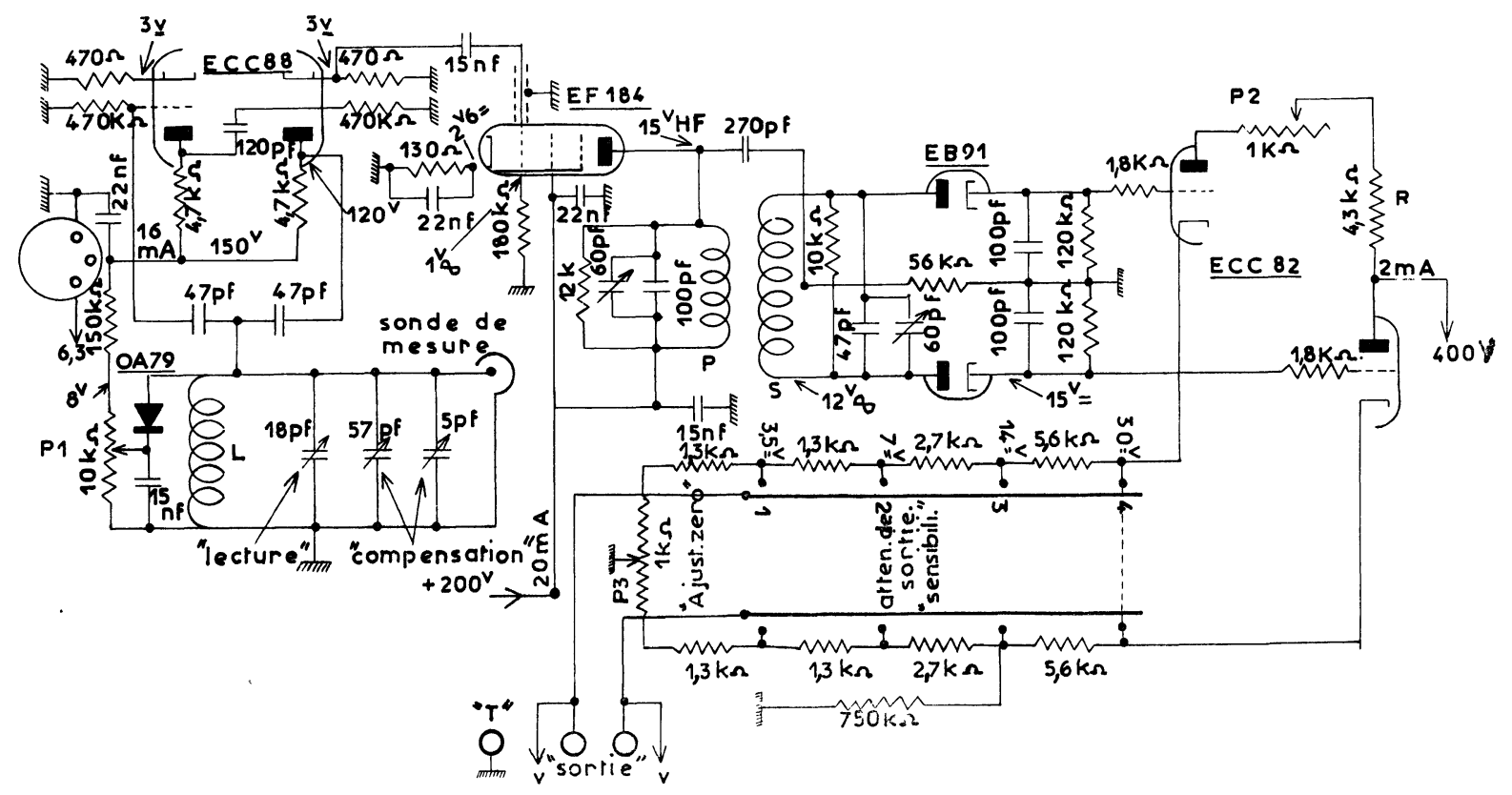

FIG. 2.

L : 20 spires $10 / 10 ; \varnothing 25$ : pas 1,5 .

$\mathrm{P}: 13$ spires $4 / 10 ; \varnothing 8$ : jointif.

$\mathrm{S}: 2 \times 12,5$ spires $10 / 10 ; \varnothing 10$ : jointif; dist. PS : $18 \mathrm{~mm}$; dist. entre 2 secondaires : $10 \mathrm{~mm}$. 


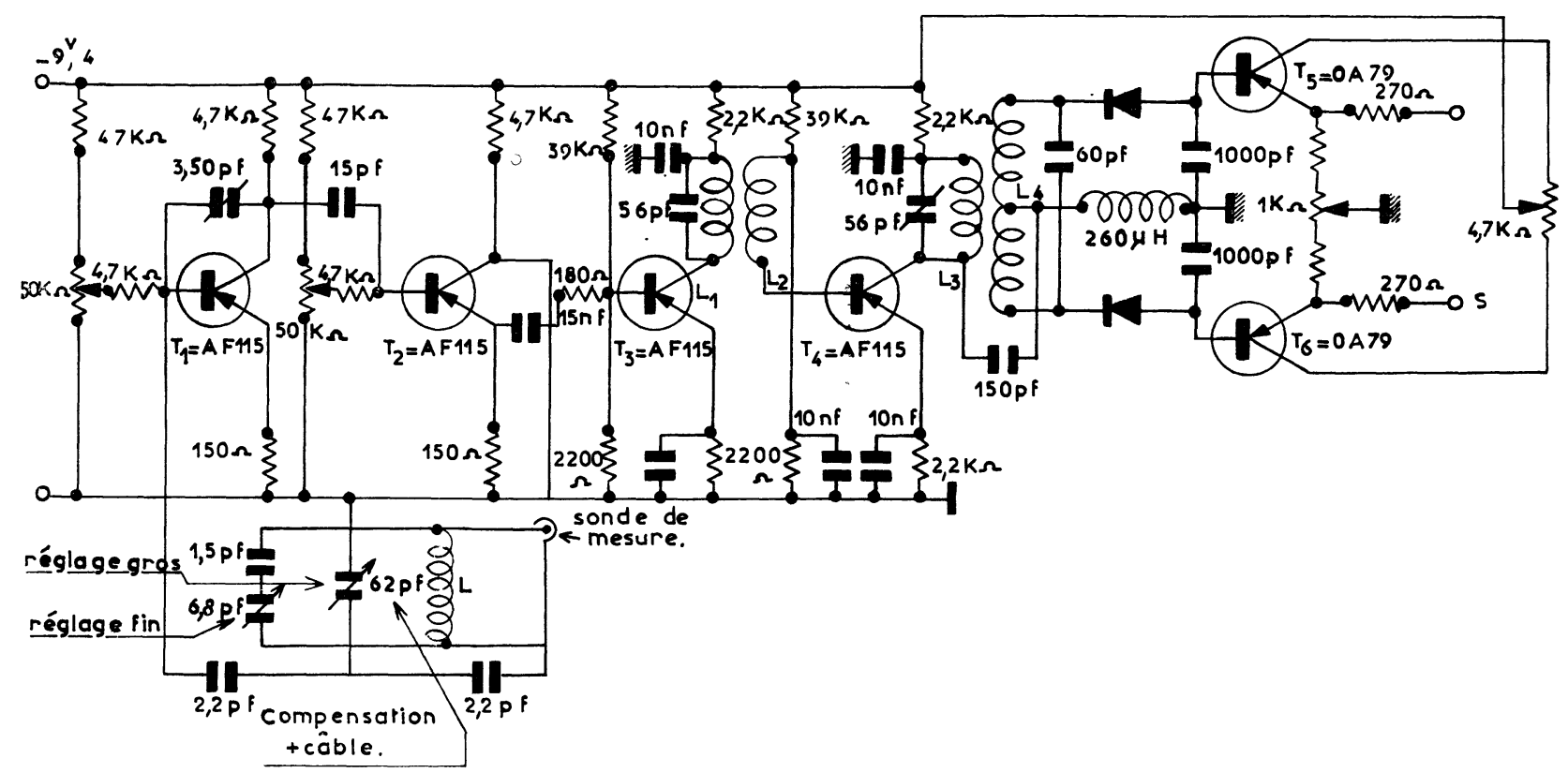

FIG. 3.

$\mathrm{L}_{1}: 4,7 \mu \mathrm{H} ; Q: 107 ; R: 2,7 \Omega ; Z \approx 30 \mathrm{k} \Omega ; 18$ spires en fil à brins divisés sur LIPA avec noyau en ferrite. $\mathrm{L}_{2}$ : bobiné à $1 \mathrm{~mm}$ de $L_{1}$ sur le même mandrin, même fil, 3 spires.

$\mathrm{L}_{3}: 4,2 \mu \mathrm{H} ; Q: 70 ; R: 4 \Omega ; Z \approx 20 \mathrm{k} \Omega ; 35$ spires sur tube de verre $\varnothing 6 \mathrm{~mm}$, sans noyau.

$\mathrm{I}_{14}: 2 \times 4,1 \mu \mathrm{H} ; Q: 78 ; 2 \times 26$ spires sur LIPA sans noyau.

$L_{1}$ : en cuivre émaillé de $8 / 10 ; 28$ spires jointives bobinées sur mandrin air (longueur $24 \mathrm{~mm}$ ) $10 \mathrm{~mm}$, monté sur un bouchon normal dans un tube de laiton étamé rempli d'araldite pour maintenir rigides les spires de la self.

avec un élément déformable sous l'influence d'une faible force ou d'une petite variation de pression différentielle. Lorsque le déplacement agit sur l'écartement des armatures, la sensibilité est telle qu'on peut utiliser un élément déformable de grande raideur et allier ainsi sensibilité et faible constante de temps. Un tel dispositif a permis à l'un de nous [9] de mesurer simultanément la force de recul liée à la sublimation sous basse pression d'un solide volatil, et la température de la substance, mettant ainsi en évidence le rôle déterminant du transfert thermique et l'action des forces radiométriques perturbatrices.

Dans d'autres applications, le phénomène agit sur la surface des armatures en regard; on peut par exemple transformer n'importe quel manomètre à mercure en appareil enregistreur sensible. Il suffit pour cela de placer sur le tube de verre deux armatures hémi-cylindriques reliées au capteur. Le déplacement du mercure dans le tube modifie le couplage capacitif entre les armatures et peut être traduit linéairement et enregistré. La sensibilité est encore accrue si l'on peut disposer d'un contact électrique dans la masse du mercure; une seule plaque de détection externe suffit alors. En limitant les vibrations mécaniques, et en tenant compte des déformations possibles du ménisque du mercure, nous avons ainsi obtenu des enregistrements d'une sensibilité et d'une fidélité compa- rables à celles des mesures au cathétomètre. Dans sa version transistorisée, l'appareil peut être rendu transportable et très commode en disposant les armatures de détection sur la face interne d'une pince qu'il suffit de placer suivant les besoins sur le tube manométrique où l'on désire suivre la pression.

Les possibilités de l'appareil apparaîtront plus clairement dans son association à une balance de torsion, que nous allons maintenant décrire :

Microbalance de torsion. - Nous nous sommes proposés d'étudier l'échange de quantité de mouvement qui résulte de la désorption ou de la fixation irréversibles de molécules gazeuses à la surface d'un solide. Le premier effet est bien connu : il se traduit par une force de " recul » dont la mesure par un dispositif de torsion permet d'accéder notamment à la tension de vapeur de composés peu volatils (méthode d'effusion de Langmuir). Le second effet a été beaucoup moins étudié. Lorsque les particules gazeuses se fixent sélectivement sur une seule face d'une plaquette plongée dans le gaz, on observe un effet de " succion » de la plaque en direction des particules actives. Nous nous sommes intéressés à la pression de succion qui apparaît lorsque des particules réactives engendrées photochimiquement viennent se fixer irréversiblement sur une cible solide déposée sur l'une 
des faces d'une mince plaquette de mica. On montre alors que la pression de «succion » [10], [11] s'exprime par :

$$
p_{\mathrm{s}}=\frac{\gamma \nu}{\mathscr{N}} \sqrt{\pi M R T / 2}
$$

où $v$ est le nombre de chocs des particules sur la cible par unité de surface et de temps, $M$ leur masse molaire, $\gamma$ le rendement de fixation et $\mathscr{N}$ le nombre d'Avogadro. Nos expériences ont porté sur la fixation d'hydrogène activé par dissociation photosensibilisée au mercure, sur une cible de trioxyde de molybdène, sous des pressions totales d'hydrogène comprises entre 0,1 et 1 atmosphère. Compte tenu du faible taux de dissociation accessible par cette méthode photochimique $\left(10^{-4}\right.$ environ) et de la valeur également très faible de $\gamma\left(10^{-4}\right.$ à $\left.10^{-5}\right)$, on peut s'attendre à des pressions de succion de l'ordre de $10^{-5}$ pascal. Pour étudier commodément ce phénomène, il faut disposer d'une balance de torsion permettant d'enregistrer en continu des couples de l'ordre de $10^{-9}$ newton $\times$ mètre. Les microbalances de torsion décrites dans la littérature peuvent se classer en deux catégories :

Dans la première, les rotations d'un fil de torsion d'une cinquantaine de microns sont détectées optiquement à l'aide d'un miroir [12], [13], [14], [15], [16]. Ces appareils ont surtout été utilisés pour la mesure par la méthode d'effusion, de tensions de vapeur nettement plus élevées que les pressions de succion que nous nous proposons d'étudier. Bien que leur sensibilité limite soit d'un ordre de grandeur convenable pour cette étude, ils se prêtent mal à l'enregistrement car les rotations les plus faibles ne peuvent être mesurées qu'au moyen d'un microscope.

Dans la seconde catégorie, on trouve des dispositifs beaucoup plus élaborés, faisant appel à des fils plus fins (5 à 10 microns) et à un asservissement pilotant une compensation électrostatique ou électromagnétique [17], [18], [19]. La balance de Humphrey et Johnston [19] permet par exemple de mesurer un couple de $10^{-13}$ newton $\times$ mètre avec une constante de temps d'une fraction de seconde.

Toutefois, ces appareils sont très fragiles, essentiellement adaptés à des mesures sous basses pressions avec des équipages mobiles de faible masse, et nécessitent un dispositif d'asservissement relativement complexe.

Disposant au laboratoire d'un capteur de déplacements sensible, nous avons préféré construire une balance de la première catégorie, en substituant à la détection optique classique une détection électrique. La sensibilité est accrue d'un facteur 10 environ, l'enregistrement est possible, mais l'appareil reste suffisamment robuste pour supporter des cibles d'une dizaine de grammes.

L'ÉQUIPAGE MobILE (fig. 4). - Les forces à mesurer, donc les déflexions, s'exercent dans un plan horizontal sur l'équipage mobile qui est par ailleurs équilibré

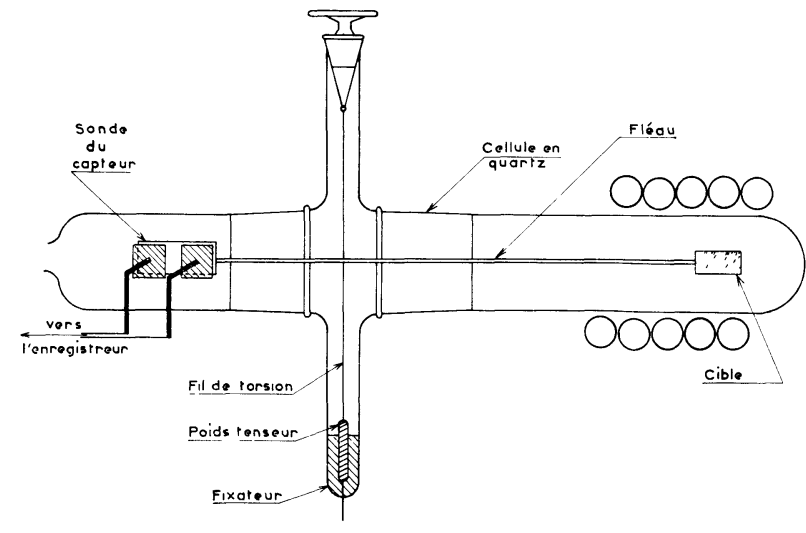

FIG. 4

Microbalance de torsion pour la mesure de la pression de succion liée à la fixation irréversible de l'hydrogène activé par photosensibilisation au mercure à la surface d'une cible en trioxyde de molybdène. Schéma de principe (voir description dans le texte).

vis-à-vis de la pesanteur. La cible est fixée à l'extrémité d'un fléau horizontal en silice de $48 \mathrm{~cm}$ de long et $1 \mathrm{~mm}$ de diamètre. Le fléau est suspendu à un fil de torsion vertical fixé à ses deux extrémités : à l'extrémité supérieure, un robinet de réglage permet d'exercer une rotation d'ajustement du zéro; à l'extrémité inférieure, une masse métallique qu'on peut immobiliser dans une cire spéciale exerce une tension convenable sur le fil et peut servir d'amenée de courant lorsqu'on veut mettre l'équipage à la masse. Le fil, de 10 à $15 \mathrm{~cm}$ de long, est soit en silice (diamètre 50 à 80 microns), soit en tungstène (diamètre 50 à $60 \mathrm{mi}$ crons).

Chacune de ces solutions présente des avantages et des inconvénients qui ont été souvent discutés [21], [22]. A l'autre extrémité du fléau, est fixée une plaque de cuivre qui exerce, lors des rotations de l'équipage, un couplage capacitif variable entre deux plaques fixes soudées à la paroi de l'enceinte. Équivalent à deux capacités variables en série, ce dispositif est particulièrement commode dans le cas des fils de silice car il ne nécessite aucune amenée de courant sur le fléau. Avec un fil en tungstène, il est possible de raccorder la plaque de cuivre à la masse et d'utiliser le capteur d'une manière classique. L'amplitude des rotations détectées doit être limitée de façon à rester dans le domaine de linéarité du capteur.

Caractéristiques statiques et Dynamiques. La constante de torsion du fil peut être estimée à partir de la période d'oscillation d'un solide de moment d'inertie connu. L'un des fils de tungstène utilisés présente une constante ainsi mesurée de $185 \pm 5 \times$ $10^{-9}$ newton $\times \mathrm{m}^{2} /$ radian. Dans ces conditions, en présence d'hydrogène sous une pression totale de 0,1 à $1 \mathrm{~atm}$, la pseudo-période d'oscillation est de $26 \mathrm{~s}$ et le facteur d'amortissement $\zeta=0,11$, ce qui corres- 
pond à un dépassement de 0,7 lors de l'application à l'équipage d'un couple susceptible de produire une rotation échelon-unité.

ÉtAlONNAGe ET LINÉARITÉ. - La constante de torsion du fil étant connue, il reste, pour étalonner la balance, à relier les déflexions du fléau au signal de sortie du capteur. A cet effet, l'équipage étant en place et le zéro fixé, on relève la tension de sortie en fonction des déplacements du fléau auquel on imprime de légères rotations statiques grâce à une jauge micrométrique de profondeur. Les figures 5 et 6 sont des exemples de tels étalonnages. Considérons l'expression de la fréquence de résonance :

$$
f=1 / 2 \pi \sqrt{L\left(C+C^{\prime}\right)} .
$$

$C$ est la capacité de la sonde, voisine de $0,5 \mathrm{pF}$, tandis que $C^{\prime}$ est de l'ordre de $60 \mathrm{pF}$. On peut donc considérer que $C \ll C^{\prime}$ et écrire, au second ordre près :

$$
f \approx \frac{1}{2 \pi \sqrt{L C^{\prime}}}\left(1-\frac{C}{2 C^{\prime}}\right) .
$$

Il en résulte pour le signal de sortie :

$$
V \sim f-f_{0} \sim \frac{e-e_{0}}{e e_{0}} \sim \frac{\Delta e}{e}
$$

où $e$ est l'écartement des plaques de détection. On peut voir sur les figures 5 ( $V$ en fonction de $\Delta e$ à $e$ constant) et $6(1 / V$ en fonction de $e$ à $\Delta e$ constant $)$ que cette relation est bien vérifiée.

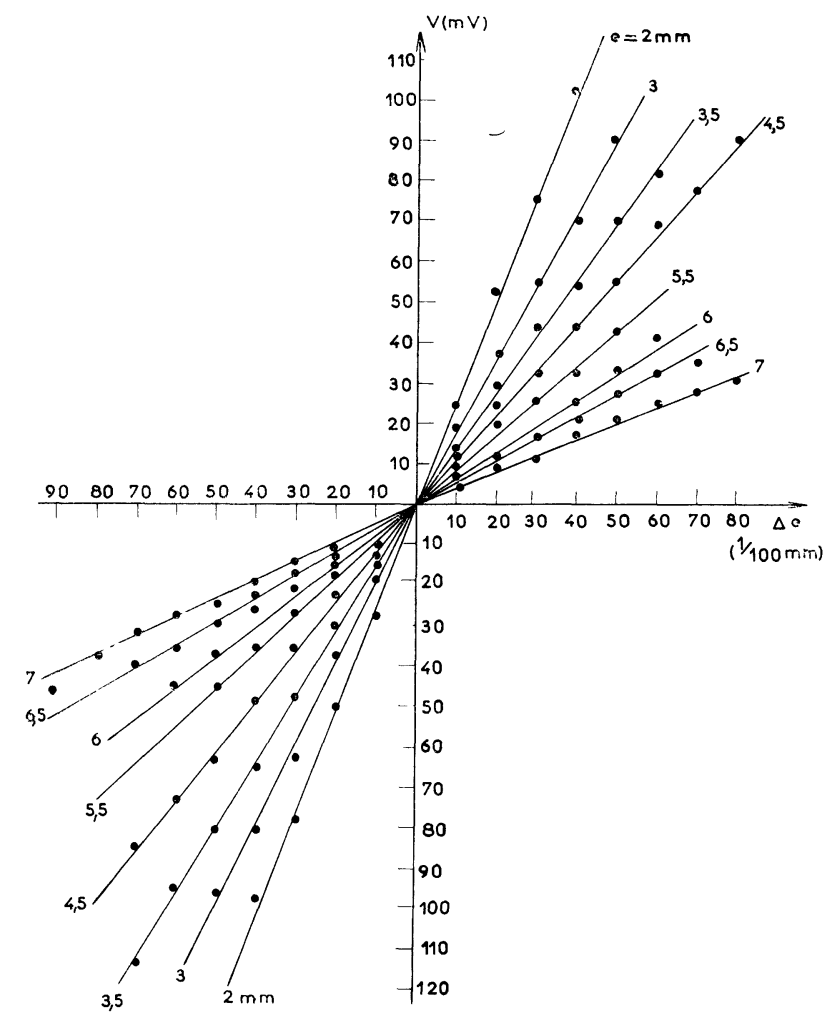

FIG. 5 .

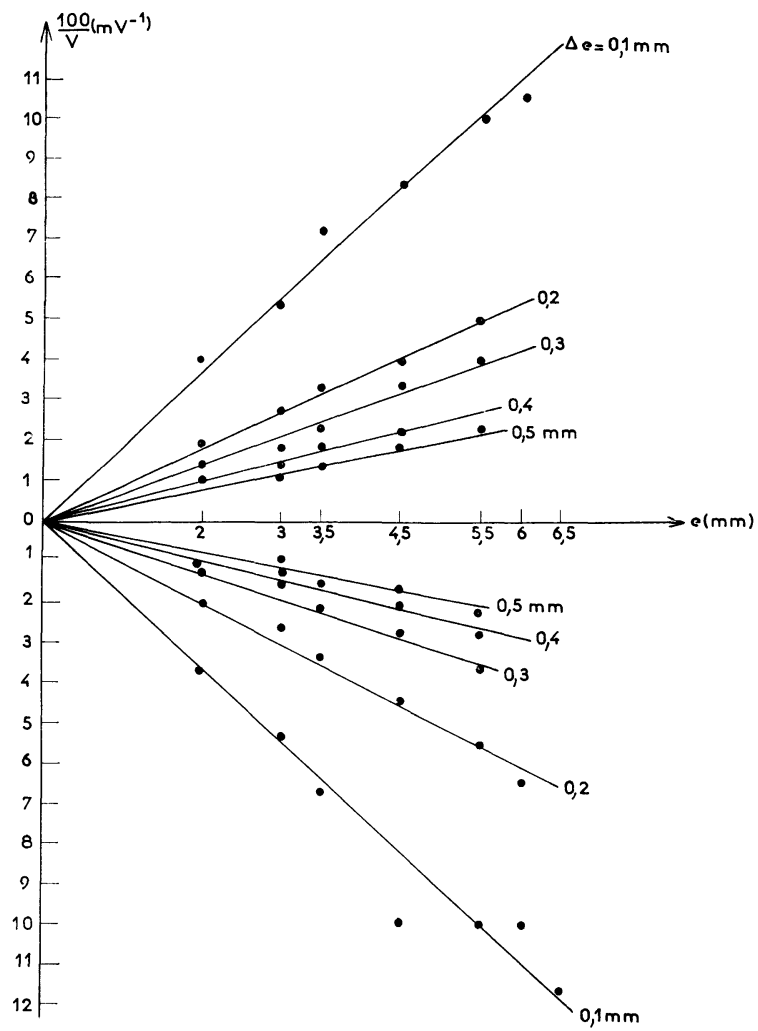

FIG. 6.

Dans ces limites d'utilisation, l'appareil est donc linéaire et sa sensibilité inversement proportionnelle à l'écartement des plaques.

Sensibilité et performances. - Finalement, on dispose d'une tension proportionnelle au couple appliqué. Par exemple, autour d'un écartement $e=1,5 \mathrm{~mm}$ et avec un fil de tungstène de 57 microns, la sensibilité est d'environ 10 millivolts pour un couple de $10^{-9}$ newton $\times$ mètre.

Compte tenu de la stabilité mécanique du système, le bruit de fond s'élève à quelques millivolts. Dans ces conditions, on apprécie encore un couple de $10^{-10}$ newton $\times$ mètre, soit une pression au niveau de la cible de $10^{-6}$ pascal, soit une rotation de $10^{\prime \prime}$ ou un déplacement relatif des armatures de 10 microns. Comme nous l'avons signalé, cette limite est nettement inférieure à celle que permet d'atteindre la détection des rotations par miroir $\left(0,01^{\circ}\right.$ dans la référence [15]).

La figure 7 montre un exemple d'enregistrement

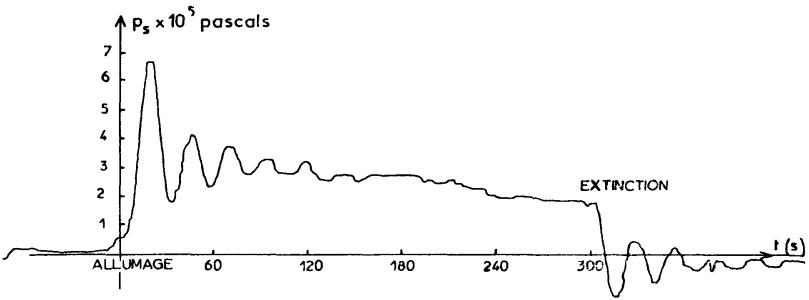

FIG. 7. 
obtenu sous une pression totale $P=76$ torrs d'hydrogène. Le phénomène de succion y est nettement mis en évidence : il se manifeste pendant la période d'allumage de la lampe à vapeur de mercure qui engendre les particules d'hydrogène actif. L'étude détaillée du phénomène est donnée ailleurs [23], [24].

Signalons seulement ici que des mesures stables et reproductibles de forces de succion nécessitent l'élimination d'un certain nombre d'effets perturbateurs :

Les vibrations mécaniques et les dérives thermiques sont limitées au maximum par l'installation de l'appareil dans un laboratoire de sous-sol à température constante. Les charges électriques localisées à la sur- face du quartz induisent des forces électrostatiques qui disparaissent au cours du temps, à condition de n'exercer ensuite sur le réacteur que des actions à distance. Des forces radiométriques, dues à l'échauffement de la cible, se manifestent dans l'hydrogène sous une pression de $10^{-2}$ à $10^{-1}$ torr. Elles se traduisent par une pression de recul qui peut atteindre $10^{-3}$ pascal : cet effet a complètement disparu au-dessus de 10 torrs, ce qui constitue une limite inférieure au domaine de pression totale qu'il est possible d'étudier. Par contre, le calcul montre que la pression de radiation exercée par la lampe sur la cible est inférieure de plusieurs ordres de grandeur à la limite de détection de l'appareil.

\section{BIBLIOGRAPHIE}

[1] FoldDari (T. L.) et LION (K. S.), Instruments and control systems, 1964, 37, 7.

[2] UTTERBACK (N. G.) et GrifFith Jr (T.), Rev. Sci. Inst., 1966, 37, 866.

[3] Beynon (J. D. E.) et CaIRns (R. B.), J. Sci. Inst., 1964, 41, 111.

[4] WAXman (M.) et Chen (W. T.), J. Research Nat. Bur. Standards, 1965, 69 C, 27.

[5] LION (K. S.), Rev. Sci. Inst., 1964, 35, 353.

[6] Cook (D. B.) et Danby (C. J.), J. Sci. Inst., 1953, 30, 238.

[7] Camani (M.) et Heinrich (F.), Helvetica Phys. Acta, 1966, 39, 567.

[8] Hackam (R.), Austin (W. E.) et Thomas (R. D.), J. Sci. Inst., 1965, 42, 344.

[9] ChÉry (D.), D.E.A. de Chimie-Physique, Nancy, 1965.

[10] Bennewitz (K.) et Neumann (W.), Z. Phys. Chem., 1930, 7 B, 246.

[11] Dauvillier (A.), C. R. Acad. Sci., 1957, 244, 150.

[12] Pratit (J. N.) et AlFred (A. T.), J. Sci. Inst., 1959, 36, 465.

[13] Rosenblatit (G. M.) et Birchenali (C. E.), $J$. Chem. Phys., 1961, 35, 788.
[14] Rosen (C. L.) et Melveger (A. J.), J. Phys. Chem., 1964, 68, 1079.

[15] Munir (Z. A.) et SEARCy (A. W.), J. Electrochem. Soc., 1964, 111, 1170.

[16] Wilson (A. E.), KIm (J. H.) et Cosgarea Jr (A.), Rev. Sci. Inst., 1965, 36, 1428.

[17] Rogalio (V. L.) et Savage (H. F.), Rev. Sci. Inst., 1963, 34, 988.

[18] Siegle (W. T.) et BeAm (W. B.), Rev. Sci. Inst., 1964, 35, 1173.

[19] Humphrey (F. B.) et Johnston (A. R.), Rev. Sci. Inst., 1963, 34, 348.

[20] Villekmaux (J.) et Gibert (R.), Bull. Soc. Chim. Fr., 1961, 543.

[21] Geary (P. J.), Torsion devices, B.S.I.R.A., London, 1960.

[22] FreEMaN (R. D.), Momentum Sensors, Oklahoma State Univ. Report, 1964.

[23] ChÉry (D.), Thèse de Doctorat de Chimie-Physique (3e cycle), Nancy, 1967.

[24] Chếry (D.) et Villermaux (J.), J. Chim. Phys. En cours de publication. 